\title{
Téoros
}

Revue de recherche en tourisme

\section{Des solutions internationales et canadiennes à la problématique du tourisme sexuel impliquant des enfants}

\section{Justin Roberge}

Volume 22, numéro 1, printemps 2003

Tourisme et sexualité

URI : https://id.erudit.org/iderudit/1071580ar

DOI : https://doi.org/10.7202/1071580ar

Aller au sommaire du numéro

Éditeur(s)

Université du Québec à Montréal

ISSN

0712-8657 (imprimé)

1923-2705 (numérique)

Découvrir la revue

Citer cet article

Roberge, J. (2003). Des solutions internationales et canadiennes à la problématique du tourisme sexuel impliquant des enfants. Téoros, 22(1), 15-21.

https://doi.org/10.7202/1071580ar d'utilisation que vous pouvez consulter en ligne. 


\section{Des solutions internationales et canadiennes à la problématique du tourisme sexuel impliquant des enfants}

\section{Justin Roberge}

'exploitation sexuelle des enfants est un phénomène qui se manifeste sous plusieurs formes : abus sexuels, prostitution, pornographie, etc. À ce sujet, deux auteurs expliquent que l'exploitation du plus faible par le plus fort est au cœur même du problème de l'exploitation sexuelle des enfants : « [...] power of male over female, adult over child, devious over naïve, rich over poor, organized (e.g. sex tour operators) over unorganized (individuals) » (Richman et Severiens, 1990 : 42-43).

Sur le plan international, il existe depuis longtemps un cadre juridique visant à protéger les enfants contre des formes spécifiques d'exploitation sexuelle (essentiellement la prostitution et la pornographie) et d'autres abus pouvant comprendre un élément d'exploitation sexuelle (le travail forcé et l'esclavage). Il va sans dire que la protection des enfants contre l'exploitation sexuelle a pris un nouveau sens depuis l'adoption de la Convention relative aux droits de l'enfant par l'Assemblée générale des Nations unies en 1989 et, plus récemment, par l'adoption du Protocole facultatif concernant la vente d'enfants, la prostitution des enfants et la pornographie mettant en scène des enfants. Par exemple, la Convention a entraîné la création de nouveaux moyens de protection pour les enfants ; c'est notamment le cas du Comité des droits de l'enfant. Cependant, il ne faut pas oublier les programmes d'action et les conférences internationales qui doivent leur existence à l'enthousiasme suscité par la Convention. En fait, les répercussions de la Convention se sont fait sentir tant sur les plans national qu'international.
Sur le plan national, par exemple, plusieurs États ont récemment intensifié leur lutte contre l'exploitation sexuelle des enfants. Au Canada, comme dans plusieurs autres pays, cette lutte est d'autant plus intense quand il est question de pornographie et de prostitution infantile. C'est d'ailleurs en 1985 que le Comité spécial d'étude de la pornographie et de la prostitution a publié un rapport proposant différentes réformes juridiques et sociales dans le but de s'attaquer à ces problèmes. Depuis, de nombreuses modifications ont été apportées au Code criminel. En conséquence, les lois sont maintenant plus sévères à l'endroit de ceux qui exploitent sexuellement des enfants et les autorités policières disposent de moyens de plus en plus diversifiés pour enquêter et faire condamner ces personnes.

Malheureusement, tous les États ne disposent pas de moyens identiques ni des mêmes ressources pour lutter contre l'exploitation sexuelle des enfants. Ainsi, d'un État à l'autre, les lois visant à protéger les enfants ne sont pas appliquées avec la même rigueur. Par conséquent, il existe des endroits où l'exploitation sexuelle des enfants est rarement réprimée ou alors réprimée, mais avec peu de sévérité. Cela a pour effet « d'inviter» (si l'on peut dire) dans ces pays des étrangers qui y voient l'occasion de commettre des actes qu'ils n'oseraient pas commettre dans leur pays d'origine. Comme l'affirme le Rapporteur spécial sur la vente d'enfants au sujet de la prostitution des enfants et de la pornographie impliquant des enfants, " [l]'extrême pauvreté et l'insuffisance du système judiciaire donnent aux pédophiles l'impression qu'ils peuvent aller [dans certains pays] et abuser sexuellement d'enfants sans risquer d'avoir à rendre compte de leurs actes devant la justice » (Rapporteuse spéciale, 1998 : par. 13). Ce phénomène est communément décrit comme du « tourisme sexuel ».

Il n'existe pas de définition juridique du tourisme sexuel sur le plan international. Toutefois, le Protocole facultatif à la Convention relative aux droits de l'enfant concernant la vente d'enfants, la prostitution des enfants et la pornographie impliquant des enfants, lorsqu'il n'était encore qu'à l'état de projet, proposait la définition suivante, laquelle n'a pas été retenue dans la version finale du document :

On entend par tourisme sexuel impliquant des enfants le tourisme organisé dans l'intention de faciliter ou réaliser [directement ou indirectement] [la vente d'enfants], [de la pornographie impliquant des enfants], la prostitution des enfants [ou toute autre pratique sexuelle illicite].

Pour sa part, l'Organisation mondiale du tourisme, dans le cadre de la Déclaration de l'OMT sur la prévention du tourisme sexuel organisé, définit le tourisme sexuel comme étant (par. 13) :

[...] les voyages organisés de l'intérieur du secteur touristique ou de l'extérieur de ce secteur mais en se servant de ses structures et de ses réseaux, avec pour objet essentiel la réalisation d'une relation sexuelle à caractère commercial entre le touriste et des habitants au lieu de destination.

Enfin, certains auteurs ont élaboré leur propre définition. Pour l'un d'eux, Margaret A. Healey, le touriste sexuel est :

[...] a tourist who travels from the industrialized or developed nations to 
a growing number of developing countries to engage in illicit sexual activity with underage victims forbidden to them under their home countries'laws (1995:1852-1854).

La difficulté à définir le tourisme sexuel d'un point de vue juridique réside dans le fait qu'il ne s'agit tout simplement pas d'un crime. Le véritable crime est l'abus sexuel, la sollicitation à la prostitution, la réalisation de matériel pornographique impliquant des enfants, etc. Le touriste sexuel (ou touriste pédophile) est le qualificatif que l'on attribue à l'auteur du crime : il va dans un pays qui n'est pas le sien pour y commettre un acte criminel avec plus de facilité, parce que les autorités de ce pays s'intéressent moins à ce type de crime, par exemple. Au Canada, le Code criminel ne définit pas le tourisme sexuel, bien qu'il y fasse référence. Il en va de même dans le cas du Protocole facultatif à la Convention relative aux droits de l'enfant, aussi surprenant que cela puisse paraître.

Plusieurs auteurs (O’Grady, 1992 : 137 142 ; Healy, 1995 : 1859 ; Richman et Severiens, 1990 : 42 ; Hodgson, 1995 : 23-24) s'accordent pour dire que l'une des principales difficultés rencontrées dans toute enquête menée sur le tourisme sexuel impliquant des enfants consiste en l'absence de données statistiques fiables et précises sur le nombre d'enfants victimes ainsi que sur le nombre et le profil des personnes qui exploitent ces enfants. Cette absence de données s'explique principalement par le caractère illégal de l'activité en question. Malgré les mises en garde concernant le peu de fiabilité des données actuellement disponibles, la doctrine en est néanmoins abondante. Un regroupement d'organismes s'est donc intéressé aux données sur l'exploitation sexuelle commerciale des enfants qui sont reproduites dans la documentation. La conclusion de cette étude n'est pas rassurante car il semble que ces données soient souvent manipulées et reproduites (Ennew et al., 1996). Voilà qui explique l'absence dans le présent texte de données statistiques sur le tourisme sexuel ; le texte repose plutôt sur la prémisse que le tourisme sexuel est un problème réel, universel et inquiétant, ce qui, d'ailleurs, n'a plus à être démontré.
S'il semble impossible d'évaluer de façon satisfaisante l'ampleur du problème, il en va tout autrement de ses causes et de ses conséquences. Les conséquences ne diffèrent pas réellement de celles subies par les enfants victimes d'exploitation sexuelle en général : santé physique et mentale défaillante, perte de dignité, d'estime personnelle et de confiance, violences physique et psychologique, etc. De plus, ces enfants sont exposés à toutes sortes de maladies transmissibles sexuellement, dont le sida. Ils évoluent dans un milieu où la drogue est omniprésente et où ils risquent fort d'y être initiés, puis conduits à commettre différentes infractions criminelles. En effet, les enfants de la prostitution s'enfoncent, malgré eux, dans un réseau clandestin où ils participent souvent à leur propre victimisation et à celle d'autrui.

Les causes du tourisme sexuel impliquant des enfants, par contre, semblent moins connues. Pourtant, elles sont probablement aussi nombreuses que les conséquences, même si la pauvreté semble être en grande partie la principale responsable. De nombreux auteurs se sont arrêtés à identifier ces causes, dont Douglas Hodgson et Margaret A. Healy. Pour Hodgson, le tourisme de masse (arrivée massive de touristes dans un pays où l'industrie touristique est en plein essor), les groupes organisés de criminels et de pédophiles et l'érosion des tabous culturels sont les principales causes du tourisme sexuel (1995 : 26-30). Healy, quant à elle, affirme que le matérialisme, la société de consommation, le défaut de faire appliquer avec suffisamment de rigueur les lois existantes, la crainte des maladies transmissibles sexuellement et, finalement, la discrimination sexuelle sont des facteurs qui contribuent à entretenir l'offre et la demande d'enfants sur le marché du sexe (1995 : 1861-1873). Tous deux ont raison.

L'auteure Margaret A. Healy et le Rapporteur spécial sur la vente d'enfants, la prostitution des enfants et la pornographie impliquant des enfants analysent le tourisme sexuel en termes d'offre et de demande, ce qui a fait naître l'idée d'étudier la problématique dans une perspective de demande, en prenant le Canada comme sujet.

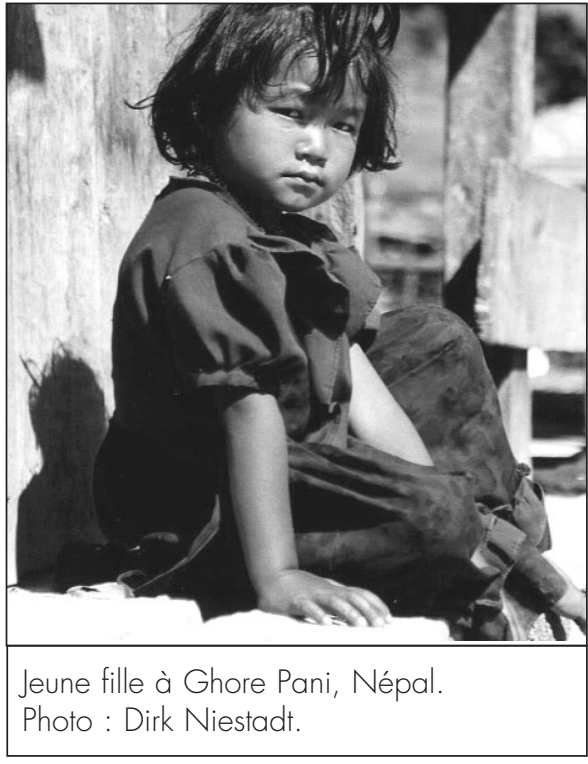

Cela implique donc une étude du tourisme sexuel du point de vue d'un État d'origine, par opposition à l'État d'accueil qui est l'État de destination des touristes sexuels. Le Canada constitue, en effet, un sujet intéressant étant donné les récentes modifications apportées au Code criminel, lesquelles permettent l'application extra-territoriale de certaines infractions du Code afin de combattre le tourisme sexuel impliquant des enfants. Une telle analyse serait toutefois incomplète si elle n'avait pas pour toile de fond le droit international. En effet, depuis le début du vingtième siècle, le droit international a développé un véritable cadre juridique afin de guider les États vers une répression efficace de certains crimes d'ordre sexuel qui sont par ailleurs étroitement liés au tourisme sexuel impliquant des enfants.

\section{Quelques instruments internationaux de protection des enfants contre le tourisme sexuel}

La Convention relative aux droits de l'enfant et son Protocole facultatif concernant la vente d'enfants, la prostitution des enfants et la pornographie mettant en scène des enfants sont, en droit international, les deux piliers de la lutte contre le tourisme sexuel impliquant des enfants. Ces instruments s'inscrivent dans un long processus d'évolution du droit international en matière de répression des crimes d'ordre sexuel. Le tableau 1 illustre l'historique du droit international 
onusien en matière de répression des crimes d'ordre sexuel au sens large :

\section{La Convention relative aux droits de l'enfant}

Issue de la Commission des droits de l'homme, la Convention relative aux droits de l'enfant 'est l'instrument juridique le plus important en matière de protection des droits des enfants. Cette convention, à laquelle le Canada est un État partie ${ }^{2}$, est le résultat combiné de l'évolution des mentalités et des perceptions envers les enfants et l'enfance et de l'évolution du droit international sur le plan de la protection des droits de la personne (Detrick, 1992 ; Freeman et Veerman, 1992). Elle constitue aujourd'hui l'instrument le plus largement ratifié : seuls la Somalie et les États-Unis ne l'ont pas ratifiée .

Avant l'avènement de la Convention, il existait deux instruments qui traitaient spécifiquement des droits des enfants : la Déclaration de Genève de 1924 et la Déclaration des droits de l'enfant de 1959. Ces deux instruments contenaient chacun une disposition portant sur l'exploitation des enfants. Le Principe 4 de la Déclaration de Genève de 1924 affirme que : "L'enfant doit être mis en mesure de gagner sa vie et doit être protégé contre toute exploitation ». Quant à la Déclaration des droits de l'enfant, elle énonce, au Principe 9 :

L'enfant doit être protégé contre toute forme de négligence, de cruau-

\begin{tabular}{|c|c|}
\hline \multicolumn{2}{|r|}{ TABLEAU 1} \\
\hline 1904 & $\begin{array}{l}\text { Arrangement international en vue d'assurer une protection efficace contre le trafic criminel connu sous le nom de } \\
\text { «traite des blanches » }\end{array}$ \\
\hline 1910 & $\begin{array}{l}\text { Convention internationale relative à la répression de la traite des blanches } \\
\text { Arrangement relatif à la répression de la circulation des publications obscènes }\end{array}$ \\
\hline 1921 & Convention internationale pour la répression de la traite des femmes et des enfants \\
\hline 1923 & Convention pour la répression de la circulation et du trafic des publications obscènes \\
\hline 1924 & Déclaration des droits de l'enfant (Déclaration de Genève) \\
\hline 1926 & Convention relative à l'esclavage \\
\hline 1948 & Déclaration universelle des droits de l'homme \\
\hline 1950 & Convention pour la répression de la traite des êtres humains et de l'exploitation de la prostitution d'autrui \\
\hline 1956 & $\begin{array}{l}\text { Convention supplémentaire relative à l'abolition de l'esclavage, de la traite des esclaves et des institutions et } \\
\text { pratiques analogues à l'esclavage }\end{array}$ \\
\hline 1959 & Déclaration des droits de l'enfant \\
\hline 1973 & Convention (no 138) de l'0lT sur l'âge minimum \\
\hline 1976 & $\begin{array}{l}\text { Pacte international relatif aux droits civils et politiques } \\
\text { Pacte international relatif aux droits économiques, sociaux et culturels }\end{array}$ \\
\hline 1981 & Convention sur l'élimination de toutes les formes de discrimination à l'égard des femmes \\
\hline 1989 & Convention relative aux droits de l'enfant \\
\hline 1990 & $\begin{array}{l}\text { Nomination par la Commission des droits de l'homme d'un Rapporteur spécial sur la vente d'enfants, la prostitution } \\
\text { des enfants et la pornographie impliquant des enfants }\end{array}$ \\
\hline 1992 & $\begin{array}{l}\text { Programme d'action pour la prévention de la vente d'enfants, de la prostitution des enfants et de la pornographie } \\
\text { impliquant des enfants }\end{array}$ \\
\hline 1994 & $\begin{array}{l}\text { Création par la Commission des droits de l'homme d'un groupe de travail en vue de rédiger un Protocole faculta } \\
\text { tif à la Convention relative aux droits de l'enfant relativement à la vente d'enfants, la prostitution des enfants et } \\
\text { la pornographie impliquant des enfants }\end{array}$ \\
\hline 1995 & $\begin{array}{l}\text { Programme d'action pour la prévention de la traite des êtres humains et de l'exploitation de la prostitution d'autrui } \\
\text { Déclaration de l'Organisation mondiale du tourisme sur la prévention du tourisme sexuel organisé }\end{array}$ \\
\hline 1996 & Congrès mondial contre l'exploitation sexuelle des enfants à des fins commerciales, à Stockholm, Suède \\
\hline 1998 & Sommet international de la jeunesse contre l'exploitation sexuelle des enfants, à Victoria, Canada \\
\hline 1999 & $\begin{array}{l}\text { Convention (no 182) de l'OIT concernant l'interdiction des pires formes de travail des enfants et l'action immédiate } \\
\text { en vue de leur élimination (et Déclaration du même nom) }\end{array}$ \\
\hline 2000 & $\begin{array}{l}\text { Protocole facultatif à la Convention relative aux droits de l'enfant, concernant la vente d'enfants, la prostitution } \\
\text { des enfants et la pornographie mettant en scène des enfants }\end{array}$ \\
\hline
\end{tabular}

té et d'exploitation. Il ne doit pas être soumis à la traite, sous quelque forme que ce soit.

L'enfant ne doit pas être admis à l'emploi avant d'avoir atteint un âge minimum approprié ; il ne doit en aucun cas être astreint ou autorisé à prendre une occupation ou un emploi qui nuise à sa santé ou à son éducation, ou qui entrave son développement physique, mental ou moral.

À l'instar des déclarations précédentes, la Convention relative aux droits de l'enfant traite de l'exploitation des enfants, mais tout en élaborant davantage. Aux termes de l'article 34 de la Convention,

Les États parties s'engagent à protéger l'enfant contre toutes les formes d'exploitation sexuelle et de violence sexuelle. À cette fin, les États prennent en particulier toutes les mesures appropriées sur les plans national, bilatéral et multilatéral pour empêcher:

a) Que des enfants ne soient incités ou contraints à se livrer à une activité sexuelle illégale;

b) Que des enfants ne soient exploités à des fins de prostitution ou autres pratiques sexuelles illégales ;

c) Que des enfants ne soient exploités aux fins de la production de spectacles ou de matériel de caractère pornographique.

En somme, l'adoption de la Convention relative aux droits de l'enfant a marqué une nouvelle et formidable étape en droit international relativement à la protection des enfants contre les crimes d'ordre sexuel. L'article 34 de la Convention traite de la problématique dans une optique plus large que ne le font les instruments relatifs à la traite des êtres humains et à l'exploitation de la prostitution d'autrui ainsi que ceux relatifs aux publications obscènes. En effet, l'article 34 ne s'applique pas à un type particulier de crime d'ordre sexuel, mais plutôt à toutes les formes d'exploitation sexuelle et de violence sexuelle. De plus, l'éventail des mesures pour empêcher l'exploitation sexuelle des enfants (mesures nationales, bilatérales, 
multilatérales) accorde aux États davantage de latitude dans leurs actions en vue de protéger les enfants. L'article 34 demeure toutefois muet sur la définition des crimes et sur les mesures concrètes que les États doivent favoriser en vue de garantir une protection appropriée aux enfants.

\section{Protocole facultatif à la Convention relative aux droits de l'enfant, concernant la vente d'enfants, la prostitution des enfants et la pornographie mettant en scène des enfants}

En 1994, la Commission des droits de l'homme a adopté la Résolution 1994/90 visant à créer un groupe de travail responsable de l'élaboration des lignes directrices d'un protocole facultatif à la Convention relative aux droits de l'enfant. En 1995, la Commission a adopté la Résolution 1995/78 visant à confier à ce même groupe la tâche d'élaborer le protocole facultatif en question. Le mandat consistait à réaliser, en collaboration avec le Rapporteur spécial sur la vente d'enfants, la prostitution des enfants et la pornographie impliquant des enfants, ainsi que le Comité sur les droits de l'enfant, un protocole facultatif à la Convention relative aux droits de l'enfant qui porterait essentiellement sur les articles 34 et 35 de cette Convention. Présidé par M. Iván Mora Godoy (Cuba), le groupe de travail a présenté plusieurs rapports sur ses activités (Projet de protocole facultatif, 1995-99), mais les divergences d'opinions entre les membres du groupe quant à la nécessité d'un tel protocole ont eu pour effet de prolonger les travaux, ce qui explique un certain retard ${ }^{4}$. Dans sa Résolution 1998/76, la Commission des droits de l'homme a toutefois exprimé son souhait de voir le projet se terminer avant le dixième anniversaire de la Convention, en 1999. Le souhait s'est réalisé, mais avec un an de retard.

Le Protocole facultatif a été adopté le 25 mai 2000 au siège des Nations unies à New York. Il est entré en vigueur le 18 janvier 2002, soit trois mois après la date du dépôt du dixième instrument de ratification. Aujourd'hui, 44 États ont ratifié le Protocole et 105 l'ont signé. Le Canada étant signataire du Protocole (signature le 10 novembre 2001), il a l'obli- gation d'agir de bonne foi dans le respect des normes qui y sont énoncées, sans toutefois être lié par celui-ci comme s'il l'avait ratifié. En effet, la signature d'un instrument juridique international témoigne de l'intention d'un État de le ratifier. L'intervalle entre la signature et la ratification permet à l'État de modifier son droit interne pour le rendre conforme aux normes internationales.

\section{L'article 2 du Protocole énonce :}

Aux fins du présent Protocole :

a) On entend par vente d'enfants tout acte ou toute transaction faisant intervenir le transfert d'un enfant ou de toute personne ou de tout groupe de personnes à une autre personne ou un autre groupe contre rémunération ou tout autre avantage ;

b) On entend par prostitution des enfants le fait d'utiliser un enfant aux fins d'activités sexuelles contre rémunération ou toute autre forme d'avantage ;

c) On entend par pornographie mettant en scène des enfants toute représentation, par quelque moyen que ce soit, d'un enfant s'adonnant à des activités sexuelles explicites, réelles ou simulées, ou toute représentation des organes sexuels d'un enfant, à des fins principalement sexuelles.

Aux termes du Protocole, la vente d'enfants est proscrite tant qu'elle concerne le fait d'offrir, de remettre ou d'accepter un enfant hors des règles établies concernant l'adoption. Le fait d'offrir, d'obtenir, de procurer ou de fournir un enfant à des fins de prostitution est également proscrit. Enfin, il en va de même du fait de produire, de distribuer, de diffuser, d'importer, d'exporter, d'offrir, de vendre ou de détenir des matériels pornographiques mettant en scène des enfants.

Les États qui ratifient le Protocole ont l'obligation de veiller à ce que ces activités soient pleinement saisies par leur droit pénal, que ces infractions soient commises au plan interne ou transnational. Il faut lire ici une référence directe au tourisme sexuel. D'ailleurs, l'introduction du Protocole énonce que les États parties sont profondément préoccupés par la pratique répandue et persistante du tourisme sexuel auquel les en- fants sont particulièrement exposés, dans la mesure où il favorise directement la vente d'enfants, la prostitution des enfants et la pornographie mettant en scène des enfants.

À la lecture du Protocole, deux éléments retiennent l'attention. D'abord, l'article 3 invite spécifiquement les États à prendre les mesures qui s'imposent afin d'établir la responsabilité des personnes morales pour les infractions mentionnées ci-haut. Cette responsabilité pourrait être pénale, civile ou administrative. Nous notons qu'il s'agit d'une recommandation plutôt que d'une obligation pour les États parties.

L'autre élément concerne l'invitation expresse qui est faite aux États parties de prendre les mesures nécessaires pour établir leur compétence aux fins de connaître les infractions ci-haut mentionnées, incluant celles commises sur le plan transnational. Or, le principe général veut que les lois pénales aient un effet territorial uniquement. Autrement dit, un État ne peut connaître que les crimes commis sur son propre territoire, d'où toute la problématique du tourisme sexuel : que faire lorsque le touriste est rentré dans son pays d'origine après avoir commis un crime ailleurs ?

L'extradition pourrait être une réponse, mais c'est sans compter le fait qu'il s'agisse d'un processus qui se déclenche à la suite d'une demande expresse formulée par un autre État. Si le manque de volonté de certains États qui profitent généreusement de l'industrie touristique est un frein à la lutte contre le tourisme sexuel, comment penser que ces mêmes États traqueront les touristes sexuels jusqu'au Canada, par exemple, en requérant leur extradition ? Le régime traditionnel basé sur la territorialité des lois et complété par l'extradition comporte donc des faiblesses en matière de lutte contre le tourisme sexuel.

Au cours d'une longue évolution, le droit international a donc élaboré quelques pistes de solution. Le Protocole les mentionne expressément. Ainsi, faisant exception au principe de la territorialité des lois, un État partie au Protocole peut connaître une infraction commise à l'extérieur de son territoire lorsque l'auteur présumé ou la victime est un ressortissant dudit État. 
En 1997, avant même que ne soient complétés les travaux sur le Protocole facultatif, le Canada a modifié son Code criminel spécifiquement pour combattre le tourisme sexuel. À l'époque, le Canada a choisi de responsabiliser ses ressortissants lorsqu'ils sont à l'étranger en permettant aux tribunaux canadiens de connaître certaines infractions criminelles d'ordre sexuel. Ce choix, fondé sur des préceptes de droit international bien établis, a été confirmé par l'adoption du Protocole facultatif.

\section{L'action canadienne contre le tourisme sexuel}

La communauté internationale n'a aucun intérêt à ce que les touristes sexuels demeurent impunis (Ticehurst, 1996 : 1826 ; Watson, 1992 : 68-69 ; Alldridge, 1997 : 31-33). Ces individus exploitent la disparité qui existe entre les différents systèmes nationaux de protection des droits de la personne dans le but d'exploiter sexuellement des enfants et de réduire les risques d'être l'objet de poursuites criminelles. Cette « double exploitation » (des enfants et des systèmes judiciaires) est un constat difficile à faire pour un système universel de protection des droits de la personne...

Les États d'origine des touristes pédophiles ont intérêt à prendre des mesures répressives contre leurs ressortissants délinquants. Tout d'abord, en les responsabilisant pour leur comportement à l'étranger, les États protègent par le fait même leurs autres ressortissants. En effet, les personnes qui commettent des actes criminels à l'étranger sont susceptibles de reproduire ces actes dans leur propre pays. L'intérêt qu'a un État d'origine à adopter des mesures répressives contre les touristes sexuels existe également quand la demande lui est faite par un État d'accueil, puisque cela permet notamment d'entretenir de bonnes relations entre les deux États. Enfin, l'intérêt est manifeste dans tous les cas où le touriste sexuel risque de demeurer impuni pour ses activités criminelles. En effet, certains États refusent d'extrader leurs ressortissants en l'absence d'un traité formel. De plus, même en présence d'un traité, l'extradition pourrait être refusée sous prétexte qu'elle aurait pour conséquence de nier au suspect certains droits fondamentaux. Enfin, dans l'éventualité où le touriste sexuel se trouvait toujours sur le territoire de l'État d'accueil, cet
État pourrait renoncer à le traduire devant un tribunal pénal par manque de ressources ou même par manque d'intérêt. Dans plusieurs cas, le touriste sexuel risque donc de demeurer impuni pour ses crimes.

À la lumière de ces faits, l'adoption d'une loi pénale à portée extra-territoriale semble être une solution souhaitable, sinon idéale, pour les États d'origine.

Au Canada, c'est le 18 avril 1996 que l'honorable Marcel Massé, au nom du ministre de la Justice et procureur général du Canada, a présenté à la Chambre des communes en première lecture le projet de loi C-27 visant notamment à combattre le tourisme sexuel impliquant des enfants. Ce projet de loi était inspiré en partie d'un projet de loi privé présenté auparavant par $\mathbf{M}^{\mathrm{me}}$ Christiane Gagnon, députée du Bloc québécois. Le 10 juin 1996, le projet de loi a été lu pour la deuxième fois puis envoyé pour examen au Comité permanent de la justice et des questions juridiques. C'est le 14 avril 1997 que la Loi modifiant le Code criminel (prostitution chez les enfants, tourisme sexuel impliquant des enfants, harcèlement criminel et mutilations d'organes génitaux féminins) a été adoptée en troisième lecture par la Chambre des communes, soit près d'un an suivant la première lecture. La loi, qui comporte un long préambule, a reçu la sanction royale le 25 avril 1997 et est entrée en vigueur le 26 mai 1997.

Le préambule de la loi témoigne de l'inquiétude du Parlement canadien face à la situation des enfants victimes de violence et plus particulièrement face à la prostitution des enfants, au Canada comme à l'étranger. Il contient de plus quelques références à des instruments juridiques internationaux et à des événements particuliers qui permettent de soutenir que les inquiétudes du Parlement canadien sont également partagées par la communauté internationale. Curieusement, les instruments et les événements mentionnés ne sont pas les plus pertinents qui existaient au moment de l'adoption de la loi.

La loi, quant à elle, prévoit :

1. L'article $7 \mathrm{du}$ Code criminel est modifié par adjonction, après le paragraphe (4), de ce qui suit :
(4.1) Nonobstant les autres dispositions de la présente loi ou toute autre loi, la personne qui, à l'étranger, commet un acte par action ou omission qui, s'il était commis au Canada, constituerait une infraction aux articles 151, 152, 153, 155 ou 159, aux paragraphes 160(2) ou (3), aux articles 163.1, 170, 171 ои 173 ои аи раragraphe 212(4) est réputée avoir commis cet acte au Canada si elle a la citoyenneté canadienne ou est un résident permanent au sens de la Loi sur l'immigration.

SECTION ABROGÉE : (4.2) Il ne peut être engagé de procédure relativement à un acte commis par action ou omission qui, s'il était commis au Canada, constituerait une infraction aux articles 151, 152, 153, 155 ou 159, aux paragraphes 160(2) ou (3) ou aux articles 163.1, 170, 171 ou 173 que si une demande est présentée au ministre de la Justice du Canada par :

a) tout fonctionnaire consulaire ou agent diplomatique accrédité auprès du Canada par l'État sur le territoire duquel l'infraction a été commise ;

b) tout ministre de cet État communiquant avec lui par l'intermédiaire des agents diplomatiques de Sa Majesté dans cet État.

(4.3) Les procédures visées au paragraphe (4.2) ne peuvent être engagées qu'avec le consentement $d u$ procureur général. [...]

En somme, tout ressortissant canadien qui commet à l'étranger un acte qui, s'il était commis au Canada, constituerait l'une des quelconques infractions sexuelles impliquant un enfant, peut être accusé et reconnu coupable par un tribunal canadien. Précisons que le paragraphe (4.2) ainsi que ses sous paragraphes a) et b) ont été abrogés en l'an 2000.

Les infractions concernées sont les suivantes : contacts sexuels (151 C.cr.), incitation à des contacts sexuels (152 C.cr.), exploitation sexuelle d'un adolescent (153 C.cr.), inceste (155 C.cr.), relations sexuelles anales (159 C.cr.), bestialité (160 (2) et (3) 
C.cr.), pornographie infantile (163.1 C.cr.), proxénétisme (170 C.cr.), maître de maison (171 C.cr.) et exhibitionnisme (173 C.cr.).

La principale motivation ayant conduit à l'adoption du projet de loi C-27 ne concernait pas le fait que les Canadiens étaient particulièrement impliqués dans le tourisme sexuel. Au contraire, bien que les réseaux de pédophiles soient actifs dans le monde entier, il semble que le Canada ne figure pas sur la liste des principaux pays d'origine. Les représentants de deux ONG (organisations non gouvernementales) ont d'ailleurs circonscrit la problématique à une plus petite échelle encore en témoignant que les touristes sexuels ne sont responsables que d'une faible proportion de tous les enfants victimes d'exploitation sexuelle commerciale. Il semble donc que la loi ait été adoptée plus pour prévenir une situation que pour en corriger une. Néanmoins, lors de la présentation du projet de loi devant la Chambre des communes pour la deuxième lecture, le ministre des Affaires étrangères a déclaré que des fonctionnaires de Revenu Canada l'avaient auparavant informé que des citoyens canadiens vont effectivement à l'étranger pour s'adonner à l'exploitation sexuelle d'enfants '. Rien de plus à ce jour.

Le ministre des Affaires étrangères et l'avocat général principal de la section de la politique en matière de droit pénal ont tous deux précisé que c'est l'effet dissuasif des mesures proposées qui importe dans la loi.

Un des sujets dont il a été question devant le Comité concernait l'application des mesures pénales à portée extra-territoriale. Ce sujet comporte deux volets : l'un concerne l'obtention des éléments de preuve et l'autre le fardeau financier que peut représenter l'application des mesures proposées par le projet de loi pour le système judiciaire canadien.

En ce qui concerne l'obtention des éléments de preuve, le Comité a tôt fait de remarquer que cela risque de devenir une tâche fastidieuse et que la coopération avec les services de police étrangers est susceptible de soulever des problèmes d'admissibilité de preuve devant les tribunaux canadiens. Un témoin a déclaré au Comité qu'il est déjà difficile d'obtenir la coopération policière en matière de trafic de drogue et de blanchiment d'argent et que, par conséquent, il est difficile de croire qu'une loi qui concerne l'exploitation sexuelle des enfants sera appliquée lorsque la plupart des pays concernés vivent du tourisme. Le représentant du ministre de la Justice, conscient de ces difficultés, a répondu que les options qui s'offrent alors consistent soit à faire venir les témoins au Canada, soit à envoyer une commission rogatoire à l'étranger. Toutefois, une réflexion sérieuse devrait précéder le choix de la première option pour s'assurer de ne pas nuire au meilleur intérêt de l'enfantvictime (Bureau international, 1998). En effet, il y a certainement un équilibre à créer entre la nécessité de punir les touristes sexuels et la nécessité d'obtenir le témoignage de l'enfant quand ce dernier doit être déplacé d'un pays à un autre pour témoigner.

Le second volet lié à l'application des mesures pénales à portée extra-territoriale, soit le fardeau financier, découle directement du problème de l'obtention des éléments de preuve. À cela, un représentant du ministère de la Justice a répondu que le Gouvernement ne prévoyait pas affecter de ressources supplémentaires aux procureurs généraux des provinces, mais que, malgré cela, rien ne permettait de croire à du ressentiment de la part des provinces, ni qu'elles n'appliqueront pas les dispositions en question. En somme, bien que tous soient conscients du fardeau financier que suppose l'adoption de mesures pénales à portée extra-territoriale, la question a rapidement été écartée. Pourtant, n'est-il pas illusoire de croire qu'aucune considération économique n'influencera la décision d'entreprendre ou non une poursuite criminelle pour des actes commis à l'étranger ?

\section{Conclusion}

La répression du tourisme sexuel par le Canada ne relève pas d'une utopie. Au contraire, cela constitue un projet réalisable et même souhaitable. Le droit international, d'une part, reconnaît une responsabilité aux États d'origine comme le Canada dans la lutte contre l'exploitation sexuelle des enfants à l'étranger par leurs ressortissants et, par conséquent, leur fournit les moyens nécessaires pour combattre cette pratique. Le Canada, d'autre part, reconnait avoir une obligation de protection envers ces enfants et, par conséquent, permet la répression d'actes sexuels commis à l'étranger par ses ressortissants.

Malgré les nombreuses critiques dont a fait l'objet le projet de loi C-27 lors de son examen par le Comité permanent de la justice et des questions juridiques, tous ont reconnu la nécessité des mesures proposées. Toutefois, plusieurs personnes auraient souhaité que la loi soit davantage répressive et rencontre moins d'obstacles dans son application, sans compter certaines incohérences difficilement explicables dont l'inclusion d'infractions relativement peu importantes parmi celles qui se voient reconnaître une portée extra-territoriale (exhibitionnisme ?). S'il est vrai qu'en matière de répression du tourisme sexuel tous les États d'origine sont en terrain inexploré, cela devrait normalement inciter le Gouvernement canadien à être plus consciencieux dans l'élaboration d'une semblable loi. L'abrogation du paragraphe (4.2) est un exemple : la loi est corrigée après son adoption avant d'avoir servi même une seule fois. Cela étant dit, l'abrogation de ce paragraphe était souhaitable car il n'aurait jamais dû exister.

Plusieurs témoins interrogés par le Comité, de même que des membres du Comité ont compris que la loi ne serait évidemment pas parfaite et ils ont alors fait de nombreuses propositions au Gouvernement dans le but d'intensifier la lutte contre le tourisme sexuel sur tous les fronts. Ainsi, plusieurs ont insisté sur la nécessité de faire connaître les dispositions de la loi à la population canadienne, de soutenir les États d'accueil, notamment sur les plans financier et diplomatique, de maintenir un engagement ferme auprès des Nations unies, de favoriser la participation de tous les intervenants, plus particulièrement des ONG, dans la lutte contre le tourisme sexuel impliquant des enfants.

Les nouvelles dispositions du Code criminel apparaissent donc comme un moyen parmi d'autres pour combattre le tourisme sexuel. Aussi, il est juste d'affirmer qu'il s'agit d'un moyen de dernier recours, valable après l'échec de la justice étrangère et du processus d'extradition. Outre l'aspect dissuasif substantiel qui est reconnu à la loi, celle-ci permettra-t-elle réellement de faire condamner des 
touristes sexuels ? La réponse est affirmative si l'on y met de la bonne volonté et suffisamment de ressources financières... Ajoutons que l'aide des ONG qui œuvrent pour la protection des droits de l'enfant dans les États d'accueil pourra certainement faire la différence, ne serait-ce que pour dénoncer les touristes sexuels ou retrouver les jeunes victimes.

\section{Justin Roberge est détenteur d'une} maîtrise en droit de l'Université de Montréal. Il est avocat et pratique le droit familial chez. Maria R. Battaglia, avocats.

\section{Notes}

1 La Convention est entrée en vigueur en droit international le 2 septembre 1990, soit le trentième jour qui a suivi la date du dépôt auprès du secrétaire général de l'Organisation des Nations unies du vingtième instrument de ratification, tel que le prévoit l'article 49 .

2 Le Canada a signé la Convention le 28 mai 1990 et l'a ratifiée le 12 décembre 1991. La Convention est entrée en vigueur au Canada le 12 janvier 1992.

3 Néanmoins, les États-Unis ont signé la Convention. Aux termes de l'article 18 de la Convention de Vienne sur le droit des Traités (1980, vol. 1155), Recueil des traités des Nations unies (p. 353), la signature d'un traité impose à l'État signataire une obligation de respect de bonne foi de l'objet et du but de l'instrument.

4 En effet, dès la première session du groupe de travail, plusieurs délégations ont fait part de leur scepticisme quant à l'utilité d'investir des ressources dans la création d'un protocole facultatif à la Convention plutôt que dans le renforcement de l'application de la Convention elle-même et des autres normes internationales. Mme Santos Pais et M. Kolosov, deux membres du Comité des droits de l'enfant, ont déclaré qu'il valait mieux éviter la prolifération des instruments de protection des droits de la personne, faisant ainsi référence aux directives émises dans la Déclaration et programme d'action de Vienne, Doc. N.U., A/CONF.157/23, qui reconnaît l'importance de maintenir la haute qualité des normes internationales en vigueur et de prévenir la prolifération des instruments relatifs aux droits de l'homme. Les membres du Comité des droits de l'enfant ont ajouté que la priorité devait être accordée à l'implantation des normes internationales existantes (Projet de protocole facultatif, 1996 : par. 48). Voir également : Cappelaere (1996).
5 Débats de la Chambre des communes (10 juin 1996) à la p. 3570 (Lloyd Axworthy, ministre des Affaires étrangères). Cette information a été répétée devant le Comité permanent de la justice et des questions juridiques : Comité permanent de la justice et des questions juridiques, témoignages, séance no 46 (1er octobre 1996), p. 1640 (Elissa Lieff, avocate-conseil, Politiques de droit pénal, ministère de la Justice).

\section{Bibliographie}

Convention relative aux droits de l'enfant, Rés. A.G. 44/25, Annexe [1992], R.T. Can. no 3 ; R.E.I.Q. (1990-92), 1992 (4), p. 361.

Déclaration de l'Organisation mondiale du tourisme sur la prévention du tourisme sexuel organisé, 11e session, A/RES/338(XI) (1995).

Déclaration des droits de l'enfant (Déclaration de Genève), S.D.N., J.O., supp. Spécial no 23, Actes de la cinquième Assemblée, séances plénières, compte-rendu des débats, Genève, 1924, p. 181.

Déclaration des droits de l'enfant, A.G. Rés. 1386 (XIV), Doc. Off. A.G., 14 e session, supp. no 16, p. 19, Doc. N.U., 1959 (Résolution A/4354) ;

Loi modifiant le Code criminel (prostitution chez les enfants, tourisme sexuel impliquant des enfants, harcèlement criminel et mutilation d'organes génitaux féminins), L.C. 1997, c. 16.

Projet de protocole facultatif à la Convention relative aux droits de l'enfant, concernant la vente d'enfants, la prostitution des enfants et la pornographie impliquant des enfants, Rapport du groupe de travail sur les travaux de sa première session : Doc. off. CES N.U., 51 ${ }^{\mathrm{e}}$ session, Doc. N.U. E/CN.4/1995/95 ; deuxième session : Doc. off. CES N.U., 52 ${ }^{\mathrm{e}}$ session, Doc. N.U. E/CN.4/1996/101 ; troisième session : Doc. off. CES N.U., 53 ession, Doc. N.U. E/CN.4/1997/97 ; quatrième session : Doc. off. CES N.U., 54 ${ }^{\mathrm{e}}$ session, Doc. N.U. E/CN.4/1998/103 ; cinquième session : Doc. off. CES N.U., 55 ${ }^{\mathrm{e}}$ session, Doc. N.U. E/CN.4/1999/74.

Rapport de la Rapporteuse spéciale sur la vente d'enfants, la prostitution des enfants et la pornographie impliquant des enfants, Mme Ofelia Calcetas-Santos, Doc. off. CES N.U., 54 session, Doc. N.U. E/CN.4/1998/101. Alldridge, Peter (1997), « The Sexual Offences (Conspiracy and Incitement) Act 1996 », Criminal Law Review, vol. 30.

Bureau international des droits des enfants (1998), La législation extraterritoriale en réponse aux dimensions internationales de l'exploitation sexuelle des enfants, Rapport des premières audiences publiques du Tribunal international des droits des enfants, mars.
Cappelaere, Geer (1996), « Possible Optional Protocols to the UN Convention on the Rights of the Child », dans Eugeen Verhellen, Monitoring Children's Rights, Martinus Nijhoff Publishers, Boston.

Detrick, Sharon (1992), The United Nations Convention on the Rights of the Child : A Guide to the "Travaux préparatoires", Dordrecht, Kluwer Academic Publishers.

Ennew, Judith, Kusum Gopal, Janet Heeran, et Heater Montgomery (1996), Children Prostitution: How Can We Measure and Monitor the Commercial Sexual Exploitation of Children? Literature Review and Annotated Bibliography, 2nd ed. edition with additional material prepared for the Congress Against the Commercial Sexual Exploitation of Children, Stockholm, 26-31 août, en ligne : Childwatch International [http://childhouse.uio.no/child watch/cwi/projects/indicators/prostitution/index. html].

Freeman, Michael, et Philip Veerman (dir.) (1992), The Ideologies of Children's Rights, Dordrecht, Kluwer Academic Publishers.

Healy, Margaret A. (1995), « Prosecuting Child Sex Tourists at Home: Do Laws in Sweden, Australia, and the United States Safeguard the Rights of Children as Mandated by International Laws? », Fordham International Law Journal, vol. 18 .

Hodgson, Douglas (1995), « Combating the Organized Sexual Exploitation of Asian Children : Recent Developments and Prospects », International Journal of Law and the Family, vol. 9.

O'Grady, Ron (1992), The Child and the Tourist, ECPAT, Auckland (N.-Z.), Pace Publishing.

Richman, Bruce, et Ruth Severiens (1990), "Child Sexual Exploitation in Developing Countries ", Review of the International Commission of Jurists, vol. 44.

Ticehurst, Rupert (1996), « Jurisdiction in 'Sex Tourism' Cases », New Law Journal, vol. 146.

Veerman, Philip (1992), The Rights of the Child and the Changing Image of Childhood, Norwell, MA, Kluwer Academic Publishers.

Verhellen, Eugeen (1996), Monitoring Children's Rights, Martinus Nijhoff Publishers, Boston.

Watson, Geoffrey R. (1992), « Offenders Abroad : The Case for Nationality-Based Criminal Jurisdiction », Yale Journal of International Law, vol. 17. 